\title{
Sickle Cell Nephropathy: Screening Provides an Opportunity to Intervene
}

\author{
Christy Cathreen Thomas ${ }^{1} \cdot$ Aditi Sinha $^{2}$ (i)
}

Received: 30 March 2021 / Accepted: 12 April 2021 / Published online: 21 April 2021

(C) Dr. K C Chaudhuri Foundation 2021

Nephropathy affects all genotypes of sickle cell disease (SCD) and has subtle manifestations at onset, usually in childhood [1]. Its diagnosis relies on screening, which reveals glomerular hyperfiltration, proteinuria, hypertension and/or reduced estimated glomerular filtration rate (eGFR). In the largest screened cohort of 410 children with SCD, the prevalence of albuminuria was $20 \%$, while decreased GFR was observed in $11 \%$ patients [1]. However, blood pressure abnormalities have not been studied closely in children with SCD, and might represent a modifiable risk factor in progression of sickle cell nephropathy.

Ambulatory blood pressure monitoring (ABPM), which allows for round-the-clock measurement of blood pressures in familiar surroundings using a portable automated device, has emerged as a recommended technique to diagnose hypertension in at-risk children and adults [2]. Advantages of ambulatory monitoring over clinic blood pressure includes higher accuracy in diagnosing and predicting hypertension, better reproducibility, ability to detect masked and white coat hypertension and abnormal nocturnal dipping, and better correlation with end-organ injury, such as left ventricular mass index, carotid intima media thickness, and arterial stiffness. Based on its utility, the 2017 recommendations of the American Academy of Pediatrics on screening and management of childhood hypertension expand indications for use of ABPM, and following recommendations of the American Heart Association, provide guidance on interpreting the findings [2]. The widespread availability of ABPM has facilitated screening for hypertension in a variety of primary and secondary forms of chronic kidney disease (CKD).

Aditi Sinha

aditisinhaaiims@gmail.com

1 Department of Pediatrics, Government Medical College, Alappuzha, Kerala, India

2 Division of Nephrology, Department of Pediatrics, All India Institute of Medical Sciences, Ansari Nagar, New Delhi 110029, India
In this issue of the Journal, Dubey et al. examined renal function and 24-h blood pressure in 30 children with SCD in comparison to matched healthy controls [3]. Compared to the latter, patients with SCD had significantly higher prevalence of hypertension, proteinuria, and glomerular hyperfiltration. While almost all patients had glomerular hyperfiltration and over half were proteinuric, only $1.4 \%$ had ambulatory hypertension and $6.6 \%$ had masked hypertension. The low prevalence of hypertension might be explained by inclusion of young patients who might not have developed kidney injury and, in absence of normative data for $<5$-y-old children, were assessed using different blood pressure thresholds [4]. Confirming previous findings [1, 5], almost two-thirds of the patients had abnormal nocturnal dipping of blood pressure, which points to secondary hypertension [2].

The impact of the study is limited chiefly by its sample size, cross-sectional design, and heterogeneity among patients based on age and disease severity and management [2]. It is unclear what proportion of patients were younger than $5 \mathrm{y}$ and how ABPM was interpreted among those between 2.5 and $5 \mathrm{y}$, for whom normative reference is not provided. The threshold for defining glomerular hyperfiltration, an eGFR of $>140 \mathrm{~mL} /$ $\min / 1.73 \mathrm{~m}^{2}$, seems generous and arbitrary given the heterogeneity of definitions in previous studies [6,7]. Further, the estimate relied on modified Schwartz formula using Jaffebased creatinine, which is prone to high bias and low precision, as compared to measured GFR or that estimated from creatinine and cystatin $\mathrm{C}$ [8]. Another limitation was the lack of estimation of renal reserve, considered a surrogate for hyperfiltration [7].

Overall, the study by Dubey et al. underscores the need for early and frequent monitoring for systemic consequences of rare diseases, as they might be amenable to medical management. Experience from a wide range of kidney diseases has confirmed the role of targeting the renin-angiotensin-aldosterone system (RAAS) in retarding progression of CKD [7]. Glomerular hyperfiltration, a known target of RAAS blockade, precedes albuminuria in SCD [6] and was the most 
common aberration in the present study. The article alerts physicians to the importance of screening for kidney injury and hypertension in patients with SCD, beginning in childhood, in order to avail an opportunity for timely interventions to retard progression of nephropathy.

\section{Declarations}

Conflict of Interest None.

\section{References}

1. McPherson Yee M, Jabbar SF, Osunkwo I, et al. Chronic kidney disease and albuminuria in children with sickle cell disease. Clin J Am Soc Nephrol. 2011;6:2628-33.

2. Macumber I. Ambulatory blood pressure monitoring in children and adolescents: a review of recent literature and new guidelines. Curr Hypertens Rep. 2017;19:96.
3. Dubey SRK, Shrivastava N, Sharma T, et al. Abnormal ambulatory blood pressure and early renal dysfunction in sickle cell disease. Indian J Pediatr. 2020. https://doi.org/10.1007/s12098-020-03558-8.

4. Varda NM, Gregoric A. Twenty-four-hour ambulatory blood pressure monitoring in infants and toddlers. Pediatr Nephrol. 2005;20: 798-802.

5. Becker AM, Goldberg JH, Henson M, et al. Blood pressure abnormalities in children with sickle cell anemia. Pediatr Blood Cancer. 2014;61:518-22.

6. Lebensburger JD, Aban I, Pernell B, et al. Hyperfiltration during early childhood precedes albuminuria in pediatric sickle cell nephropathy. Am J Hematol. 2019;94:417-23.

7. Helal I, Fick-Brosnahan GM, Reed-Gitomer B, Schrier RW. Glomerular hyperfiltration: Definitions, mechanisms and clinical implications. Nat Rev Nephrol. 2012;8:293-300.

8. Lebensburger JD, Gossett J, Zahr R, et al. High bias and low precision for estimated versus measured glomerular filtration rate in pediatric sickle cell anemia. Haematologica. 2021;106:295-8.

Publisher's Note Springer Nature remains neutral with regard to jurisdictional claims in published maps and institutional affiliations. 